\title{
CONTINUOUS TRANSLATION OF HÖLDER AND LIPSCHITZ FUNCTIONS
}

\author{
H. MIRKIL
}

All functions will be complex, periodic, integrable (on $[0,2 \pi]$ ) functions of a real variable $x$. Moreover, we shall require that every function have mean zero on $[0,2 \pi]$, so that in particular non-zero constants are excluded.

\section{Plessner's characterization of absolutely continuous functions.}

An old theorem of Plessner (4), generalized to arbitrary compact groups by Bochner (1), can be taken as our starting point. Consider the functions $f$ of bounded variation on $[J, 2 \pi]$. These $f$ form a Banach space $F$ when each $f$ is normed by its total variation on $[0,2 \pi]$. And translations define a natural one-parameter group of isometries on $F$. But there exist $f \in F$ such that the translate $T_{x} f$, defined by $\left(T_{x} f\right)(y)=f(x+y)$, does not vary continuously with $x$. In fact Plessner proves that the mapping $x \rightarrow T_{x} f$ is continuous precisely when $f$ is absolutely continuous. These $f$ constitute a closed invariant subspace $\mathscr{C} F$ of $F$, and the $F$ norm can be reinterpreted on $\mathscr{C} F$ as

$$
\int_{0}^{2 \pi}\left|f^{\prime}(x)\right| d x
$$

The above result suggests a whole class of concrete "Plessner problems": one for each (non-continuous) representation $x \rightarrow T_{x}$ of a locally compact group $X$ on a Banach space $F$. Namely, to identify the $f \in F$ for which the mapping $x \rightarrow T_{x} f$ is continuous. Or equivalently, to find the largest invariant subspace $\mathscr{C} F \subseteq F$ on which the representation $x \rightarrow T_{x}$ is continuous.

Here we shall study only a small subclass of Plessner problems, in which $X$ is the reals $\bmod 2 \pi$, and $F$ is some translation-invariant Banach space of (complex, periodic, integrable) functions. Thus $F$ will always be a subspace of $L_{1}[0,2 \pi]$. We call such an $F$ a translating space if its norm obeys, for each $f \in F$,

$$
\left|\int_{0}^{2 \pi} f(x) e^{i k x} d x\right| \leqslant \text { const }\|f\| .
$$

(By 3.5 below, the $F$ norm must then be larger than the $L_{1}$ norm.) The Plessner problem is now to find the set $\mathscr{C} F$ of all continuously translating functions in $F$, an $f \in F$ being said to translate continuously if the mapping $x \rightarrow T_{x} f$ is continuous from $X$ into the Banach space $F$. It is enough to know that this mapping is continuous at a single $x_{0}$, or that the numerical function

Received August 10, 1959.

Research supported by U.S. Air Force Office of Scientific Research under Contract $49(638)-294$. 
$x \rightarrow\left\|T_{x} f-f\right\|$ is continuous at 0 , or that for each $\phi$ in the dual space $F^{*}$ the numerical function $x \rightarrow \phi\left(T_{x} f\right)$ is continuous.

(The above choice of $L_{1}$ as "universal space" is more from convenience than necessity. For some purposes it might be useful to take the space $M$ of measures, or even some larger space of Schwartz distributions. But $L_{1}$ is well suited to our present aims.)

With many classical function spaces $F$, the identification of $\mathscr{C} F$ is easy. For instance, let $L_{p}$ be the $p$ th-power-integrable functions, let $L_{\infty}$ be the bounded functions, let $C$ be the continuous (periodic) functions, and let $M$ be the Radon measures on $[0,2 \pi]$. Then for $1<p<\infty$ we have $\mathscr{C} L_{p}=L_{p}$; we have $\mathscr{C} L_{\infty}=C$; and we have $\mathscr{C} M=L_{1}$. This last fact is simply the differentiated version of Plessner's original theorem.

\section{Definition of Lipschitz spaces. The Hardy-Littlewood theorems}

for $\boldsymbol{L}_{p}$. In this paper we propose to solve the Plessner problem associated with various spaces of Hölder functions. We aim at an abstract Banach space formulation just general enough to include the Hardy-Littlewood results (3) on integration of $L_{p}$ spaces. Since the Lipschitz functions (Hölder functions of $\operatorname{order} \alpha=1$ ) require special treatment, we shall take up this case separately first.

Given a translating space $F$, with norm $\|f\|$, let us define for each $f \in F$ the associated Lipschitz seminorm

$$
\|f\|^{(1)}=\sup _{x>0} \frac{\left\|T_{x} f-f\right\|}{x} .
$$

(Since $f$ is periodic, and $1 / x$ is decreasing, the sup over $x: 0<x<\infty$ equals the sup over $x: 0<x \leqslant 2 \pi$. We define "seminorm" in such a way that it may take the value $\infty$, but never the value 0 unless $f \equiv 0$.) The $f$ for which $\|f\|^{(1)}<\infty$ constitute a subspace of $F$, which we shall call $\wedge F$, and $\|f\|^{(1)}$ defines a genuine norm on $\wedge F$. Hence we may say that $\wedge F$ is defined and normed by $\|f\|^{(1)}<\infty$.

Example 2.1. Let $F$ be the space $C$ of (periodic) continuous functions, with norm $\|f\|_{\infty}=\sup |f(x)|$. Then $\wedge C$ is the space of ordinary (periodic) Lipschitz functions, and the norm $\|f\|_{\infty}{ }^{(1)}$ of $f \in \wedge C$ is its smallest Lipschitz constant,

$$
\|f\|_{\infty}^{(1)}=\sup _{x \neq y}\left|\frac{f(x)-f(y)}{x-y}\right| .
$$

Notice that although $C<L_{\infty}$, nonetheless for the corresponding Lipschitz spaces we have $\wedge C=\wedge L_{\infty}$. Every ordinary Lipschitz function $f$ (is absolutely continuous and) has a bounded derivative $f^{\prime}$, and conversely the indefinite integral of a bounded function is Lipschitz. (We say the indefinite integral because the constant of integration is uniquely determined by our standing requirement that all functions have mean zero.) Furthermore, an easy com- 
putation shows that $\|f\|_{\infty}{ }^{(1)}=\sup _{x}\left|f^{\prime}(x)\right|$. Hence $\left\|T_{x} f-f\right\|_{\infty}{ }^{(1)}=\left\|T_{x} f^{\prime}-f^{\prime}\right\|_{\infty}$, and the latter goes to 0 with $x$ if and only if $f^{\prime}$ is (uniformly) continuous. Thus we have

THEOREM. In order that an ordinary Lipschitz function $f$ translate continuously in the Lipschitz norm it is necessary and sufficient that $f$ have a continuous derivative.

Example 2.2. For $1<p<\infty, \Lambda L_{p}$ is the space defined and normed by

$$
\|f\|_{p}^{(1)}=\sup _{x>0} \frac{\left\|T_{x} f-f\right\|_{p}}{x}=\sup _{x>0} \frac{1}{x}\left(\int_{0}^{2 \pi}|f(x+y)-f(y)|^{p} d y\right)^{1 / p} .
$$

THeOREM. $\Lambda L_{p}$ consists precisely of all indefinite integrals $\int f$ of the $f \in L_{p}$. And $\|f\|_{p}^{(1)}=\left\|f^{\prime}\right\|_{p}$. In particular, every $f \in \Lambda L_{p}$ translates continuously in $\Lambda L_{p}$.

Proof. Hardy and Littlewood (3 Theorem 22, p. 596, Theorem 24, p. 599.)

Example 2.3. The space $\Lambda L_{1}$ is defined and normed by

$$
\|f\|_{1}^{(1)}=\sup _{x>0} \frac{1}{x} \int_{0}^{2 \pi}|f(x+y)-f(y)| d y .
$$

THeorem. $\Lambda L_{1}$ is precisely the functions of bounded variation. And $\|f\|_{1}{ }^{(1)}=$ ttl. vrn. $(f)$ In order that $f \in \Lambda L_{1}$ translate continuously in $\Lambda L_{1}$ it is necessary and sufficient that $f$ be absolutely continuous.

Proof. Hardy and Littlewood (3).

Thus the Hardy-Littlewood theorems together describe the "indefinite integral spaces" $\int L_{p}$ of all the $L_{p}$ spaces, $1 \leqslant p \leqslant \infty$. We have no idea how to describe $\int F$ when $F$ is an arbitrary translating space. But when the $f \in F$ all translate continuously in $F$, then we are able to prove (Theorem 4.11 below) that $\int F$ is always the continuously translating part of $\Lambda F$.

3. Fundamental properties of translating spaces. It will be useful at this point to assemble some general facts about continuous translation. None of the results below are new. Most of them appear in (2) and (5).

Throughout this section, $F$ will be a translating space, with norm $\|f\|$.

Lemma 3.1. Each translation $T_{x}$ is a bounded operator $F \rightarrow F$.

Proof. We use the closed graph theorem. Suppose $f_{n} \rightarrow 0$ in $F$ and $T_{x} f_{n} \rightarrow g$. We must prove $g=0$. Because $F$ is a translating space, then

$$
\begin{aligned}
\int_{0}^{2 \pi} g(y) e^{i k y} d y & =\lim _{n} \int_{0}^{2 \pi}\left(T_{x} f_{n}\right)(y) e^{i k y} d y=\lim _{n} \int_{0}^{2 \pi} f_{n}(y) e^{i k(y-x)} d y \\
& =e^{-i k x} \lim _{n} \int_{0}^{2 \pi} f_{n}(y) e^{i k y} d y=0 .
\end{aligned}
$$

Hence $g$ has all Fourier coefficients zero, and the lemma is proved. 
Notice that we have not established a uniform bound on the set of all $T_{x}$.

Lemma 3.2. The set $\mathscr{C} F$ of all continuously translating $f \in F$ is a closed subspace of $F$.

Proof. Silov (5, p. 5) uses a category argument. (But if a uniform bound is assumed on the set of all translations $T_{x}$, then an elementary computation with the norm can be used instead.)

Lemma 3.3. There exists an equivalent norm on $\mathscr{C} F$ that makes all translations isometric.

Proof. Silov (5, p. 5).

Lemma 3.4. If $F$ and $G$ are translating spaces, with $F \subseteq G$, then $\|f\|_{F} \geqslant$ const $\|f\|_{G}$ for every $f \in F$.

Proof. The above norm inequality amounts to asserting that the injection $F \rightarrow G$ is continuous. We use the closed graph theorem. Suppose that $f_{n} \rightarrow 0$ in the space $F$, and that $f_{n} \rightarrow g$ in the space $G$. Examine the Fourier coefficient

$$
\gamma_{k}(g)=\frac{1}{2 \pi} \int_{0}^{2 \pi} g(x) e^{-i k x} d x .
$$

Because $G$ is a translating space, then by definition $\gamma_{k}(g)=\lim _{n} \gamma_{k}\left(f_{n}\right)$. But because $F$ is a translating space, then $\lim _{n} \gamma_{k}(f)=\gamma_{k}(0)=0$. Hence $\gamma_{k}(g)=$ 0 for all $k$, and by the uniqueness theorem for Fourier series, $g=0$. Thus $F \rightarrow G$ is continuous, and the lemma is proved.

COROLlaRy 3.5. The norm on a translating space $F$ must satisfy

$$
\|f\| \geqslant \text { const } \int_{0}^{2 \pi}|f(x)| d x .
$$

Proof. By having required all functions to be integrable, we have required $F \subseteq L_{1}$.

The corollary below is conceptually of the greatest importance, showing as it does that there is only one correct notion of convergence for the functions in a given translating space.

COROllary 3.6. Let $F$ be a translation-invariant space of (periodic, integrable) functions. Then, among all norms $\|f\|$ larger than the $L_{1}$ norm, there exists at most one (up to equivalence) that will make F complete.

Proof. Immediate from 3.4 .

Lemma 3.7. If an exponential monomial $e^{i k x}$ appears (with non-zero coefficient) in the Fourier series of some $f \in F$, then $e^{i k x}$ itself belongs to $F$.

Proof. Silov (5, p. 12).

Let $P$ be the set of all trigonometric polynomials $\sum c_{k} e^{i k x}$ (only finitely many $\left.c_{k} \neq 0\right)$. 
Theorem 3.8. The continuously translating subspace $\mathscr{C} F$ is precisely the closure in $F$ of $P \cap F$.

Proof. Silov (5, pp. 9 and 13).

Lemma 3.9. Let $F$ and $G$ be translating spaces. Then in order that $\mathscr{C} F \subseteq \mathscr{C} G$ it is necessary and sufficient that the following two conditions obtain:

(1) $\quad F \cap P \subseteq G \cap P$

(2) $\|p\|_{F} \geqslant$ const $\|p\|_{G}$, for each $p \in F$.

Proof. Since $F \cap P=(\mathscr{C} F) \cap P$, and similarly for $G$, the necessity of (1) is obvious. The necessity of (2) follows from Lemma 3.4 above.

Conversely, suppose (1) and (2), and let $f \in \mathscr{C} F$. Then there are trigonometric polynomials $p_{n} \rightarrow f$ in the $F$ norm. Since the sequence $\left\{p_{n}\right\}$ is Cauchy in $F$, then by (2) it is also Cauchy in $G$, hence $p_{n} \rightarrow g \in G$ in the $G$ norm. By Theorem 3.8, actually $g \in \mathscr{C} G$. Finally, we recall that, by our definition of translating spaces, also $p_{n} \rightarrow f$ in $L_{1}$ and $p_{n} \rightarrow g$ in $L_{1}$. Hence $f=g$, that is, $f \in \mathscr{C} G$.

Corollary 3.10. If $F \cap P=G \cap P$, with $\|p\|_{F}=\|p\|_{G}$, then $\mathscr{C} F=$ $\mathscr{C} G$, and conversely.

\section{Characterization of continuously translating Lipschitz functions} $(\alpha=1)$. This section will be devoted to the proof of Theorem 4.11 and its ancillary lemmas. Throughout, $F$ will be a translation space, with norm $\|f\|$.

Lemma 4.1. If $f \in \mathscr{C}$ F has mean

$$
\int_{0}^{2 \pi} f(x) d x=0
$$

(as it always does in this paper), then also the Banach-valued function $x \rightarrow T_{x} f$ has mean

$$
\int_{0}^{2 \pi} T_{x} f d x=0 .
$$

Proof. write $\gamma_{k}$ for the Fourier coefficient functional defined by

$$
\gamma_{k}(f)=\int_{0}^{2 \pi} f(y) e^{-i k y} d y .
$$

Let

$$
g=\int_{0}^{2 \pi} T_{x} f d x
$$

It is enough to show that $\gamma_{k}(g)=0$ for all $k$. But 


$$
\begin{aligned}
\gamma_{k}(g) & =\gamma_{k}\left(\int_{0}^{2 \pi} T_{x} f d x\right)=\int_{0}^{2 \pi} \gamma_{k}\left(T_{x} f\right) d x \\
& =\int_{0}^{2 \pi} \int_{0}^{2 \pi} f(x+y) e^{-i k y} d y d x \\
& =\int_{0}^{2 \pi} \int_{0}^{2 \pi} f(y) e^{-i k(y-x)} d y d x \\
& =\int_{0}^{2 \pi} e^{i k x} d x \int_{0}^{2 \pi} f(y) e^{-i k y} d y \\
& =\gamma_{-k}(1) \gamma_{k}(f) .
\end{aligned}
$$

Now if $k \neq 0$, then $\gamma_{-k}(1)=0$. And if $k=0$, then $\gamma_{k}(f)=0$.

Theorem 4.2. If $f \in \mathscr{C} F$, then

$$
\|f\| \leqslant \sup _{x>0}\left\|T_{x} f-f\right\| .
$$

Proof. We have

$$
\begin{aligned}
\sup _{x}\left\|T_{x} f-f\right\| & \geqslant \frac{1}{2 \pi} \int_{0}^{2 \pi}\left\|T_{x} f-f\right\| d x \\
& \geqslant|| \frac{1}{2 \pi} \int_{0}^{2 \pi}\left(T_{x} f-f\right) d x|| \\
& =||\left(\frac{1}{2 \pi} \int_{0}^{2 \pi} T_{x} f d x\right)-f||=\|f\|,
\end{aligned}
$$

the last equality by 4.1 above.

The idea used in the above proof can be made to yield a somewhat more delicate result. Namely, assume only that $f \in L_{1}$, but that each $T_{x} f-f$ belongs to $F$ and that $\left\|T_{x} f-f\right\|$ approaches 0 with $x$. Then necessarily $f \in F$. (Question: Is it enough to assume $T_{x} f-f$ belongs to $F$ for small $x$ ?)

LEMMA 4.3. $\Lambda F=\Lambda \mathscr{C} F$.

Proof. $\Lambda F=\left\{f \in F:\|f\|^{(1)}<\infty\right\}$. And $\Lambda \mathscr{C} F=\left\{f \in \mathscr{C} F:\|f\|^{(1)}<\infty\right\}$. We need only prove that $\Lambda F \subseteq \mathscr{C} F$. But if $f \in \Lambda F$, then $\left\|T_{x} f-f\right\|=O(x)$, hence

$$
\lim _{x \rightarrow 0}\left\|T_{x} f-f\right\|=0
$$

LEMMA 4.4 .

$$
\|f\|^{(1)} \geqslant \frac{1}{2 \pi}\|f\|
$$

Proof. Immediate from 4.2 , since by 4.3 we need only consider $f \in \mathscr{C} F$.

Theorem 4.5. The space $\Lambda F$ is complete for the norm $\|f\|^{(1)}$. 
Proof. Write

$$
A_{x}=\frac{T_{x}-I}{x},
$$

so that $\|f\|^{(1)}=\sup _{x}\left\|A_{x} f\right\|$. Let $\left\{f_{n}\right\}$ be a Cauchy sequence in the normed space $\Lambda F$. By 4.4, $\left\{f_{n}\right\}$ is also Cauchy in the space $F$, hence $f_{n}$ converges in $F$ to some $f \in F$.

We claim that actually $f \in \Lambda F$. For since $\left\{f_{n}\right\}$ is Cauchy in $\Lambda F$, then $\left\{f_{n}\right\}$ is bounded in $\Lambda F$, hence there is some $M<\infty$ such that $\left\|A_{x} f_{n}\right\|_{F} \leqslant M$ for all $x$ and for all $n$. Fixing $x$ and letting $n$ go to $\infty$ we see also that $\left\|A_{x} f\right\| \leqslant M$ for all $x$. That is to say, $\|f\|^{(1)} \leqslant M$, and in particular, $f \in \Lambda F$.

We claim finally that $\left\{f_{n}\right\}$ converges to $f$ in the space $\Lambda F$. By considering instead the sequence $\left\{f_{n}-f\right\}$ and changing notation, we can suppose that $f=0$. Then given $\epsilon>0$ we must find $N$ such that $\left.\left\|f_{n}\right\|\right|^{(1)} \leqslant \epsilon$ for all $n \geqslant N$. Or equivalently, we want $\left\|A_{x} f_{n}\right\| \leqslant \epsilon$ for all $n \geqslant N$ and all $x$. Because $\left\{f_{n}\right\}$ is Cauchy in $\Lambda F$, we can find $N$ such that $\left\|A_{x}\left(f_{n}-f_{m}\right)\right\| \leqslant \epsilon$ for all $n, m \geqslant N$ and all $x$. Moreover, because each $A_{x}$ is continuous $F \rightarrow F$, we can fix $x$ and $n$ and let $m$ go to $\infty$, obtaining $\left\|A_{x} f_{n}\right\|_{F} \leqslant \epsilon$ for all $n \geqslant N$ and all $x$. Hence the completeness of $\Lambda F$ is established.

Let us remark in passing that any family of operators $A_{x}$ on an arbitrary Banach space $F$ (each $A_{x}$ bounded, but no uniform bound assumed on all $A_{x}$ ) can be used to define a new norm $\sup _{x} \| A_{x} f \mid$. And exactly as in Theorem 4.5 above, we see that the associated subspace $G$ is complete for the new norm if $\sup _{x}|| A_{x} f \|_{F}>$ const $\|f\|_{F}$. Furthermore this last, seemingly contrived, condition is actually necessary for completeness of the new space $G$ (assuming that when $f \neq 0$ then at least one $A_{x} f \neq 0$ ). To prove this necessity, we appeal to the closed graph theorem. Suppose $f_{n} \rightarrow 0$ in the new (complete) $G$, and $f_{n} \rightarrow f \in F$ in the old space $F$. We want to show that $f=0$. Because $f_{n} \rightarrow 0$ in $G$, then for each $\epsilon>0$ we can find $N$ such that for $n \geqslant N$ and for all $x\left\|A_{x} f_{n}\right\|_{F}<\epsilon$. And because $f_{n} \rightarrow f$ in $F$, then $\left.\left\|A_{x} f\right\|\right|_{F}=\lim _{n}\left\|A_{x} f_{n}\right\|_{F}<\epsilon$. But $\epsilon$ was arbitrary, and hence $\left\|A_{x} f\right\|_{F}=0$ for all $x$, that is, $f=0$. Thus we see that the injection $G \rightarrow F$ is continuous and $\|f\|_{G} \geqslant$ const $\|f\|_{F}$.

Lemma 4.6. The trigonometric polynomial $p$ belongs to $F$ if and only if its derivative $p^{\prime}$ belongs to $F$.

Proof. By 3.7 it is enough to look at single exponentials $e^{i k x}$, and for these the Lemma is clearly true.

Lemma 4.7. If $p \in P \cap F$, then

$$
\frac{\left\|T_{x} p-p\right\|}{x}
$$

converges to $\left\|p^{\prime}\right\|$.

Proof. Since all vector space topologies on a finite-dimensional space are equivalent, then 


$$
\frac{T_{x} p-p}{x}
$$

converges to $p^{\prime}$ in the $F$ norm restricted to the finite-dimensional space $P_{N}$ generated by the exponential monomials $e^{i k x}$ !that actually appear in the expression $p(x)=\sum c_{k} e^{i k x}$. And then

$$
\frac{\left\|T_{x} p-p\right\|}{x}
$$

converges to $\left\|p^{\prime}\right\|$ because the norm $\|f\|$ in a normed space varies continuously with $f$.

Lemma 4.8. If $\Phi$ is a differentiable mapping from the interval $[0, x]$ into a Banach space, then

$$
\frac{\|\Phi(x)-\Phi(0)\|}{x} \leqslant \sup _{0 \leqslant \xi \leqslant x}\left\|\Phi^{\prime}(\xi)\right\| .
$$

Proof. It is enough to prove that for an arbitrary continuous functional $\Psi$

$$
\frac{|\Psi(\Phi(x)-\Phi(0))|}{x} \leqslant \sup _{\xi}\left|\Psi\left(\Phi^{\prime}(\xi)\right)\right| .
$$

But $f(\xi)=\Psi(\Phi(\xi))$ is a numerical differentiable function, and the above inequality is then a consequence of the elementary mean value theorem.

In order to state the following lemma succinctly, for any translating space $F$ let us define $\int F$ to be the set of all indefinite integrals $\int f$ of all the $f \in F$. Since $f \rightarrow \int f$ is a one-one linear mapping, commuting with all translations $T_{x}$, we can make $\int F$ a translating Banach space by transporting to it the $F$ norm, so that

$$
\|g\| S_{F}=\left\|g^{\prime}\right\|_{F}
$$

LEMma 4.9.

$$
P \cap\left(\int \mathscr{C} F\right)=P \cap(\mathscr{C} \Lambda F)=P \cap F
$$

Proof. $P \cap\left(\int \mathscr{C} F\right)=P \cap(\mathscr{C} F)$ by Lemma 4.6, and $P \cap(\mathscr{C} F)=$ $P \cap F$ by Theorem 3.8. On the other hand, $P \cap(\mathscr{C} \Lambda F)=P \cap(\Lambda F)$ again by Theorem 3.8. Clearly $P \cap(\Lambda F) \subseteq P \cap F$. The opposite inclusion will follow from Lemma 4.6 together with the second norm inequality below.

Lemma 4.10. For every $p \in P \cap F$,

$$
\left\|p^{\prime}\right\| \leqslant \frac{\left\|T_{x} p-p\right\|}{x} \leqslant \text { const }\left\|p^{\prime}\right\| .
$$

Proof. The first inequality is an immediate consequence of Lemma 4.7 . The second inequality uses Lemma 4.8, as follows. Set $\Phi(x)=T_{x} p$, so that $\Phi(0)=p$ and $\Phi^{\prime}(\xi)=T_{\xi} p^{\prime}$. Then 


$$
\begin{aligned}
\frac{\left\|T_{x} p-p\right\|}{x}=\frac{\|\Phi(x)-\Phi(0)\|}{x} & \leqslant \sup _{0 \leqslant \xi \leqslant x}\left\|\Phi^{\prime}(\xi)\right\| \\
& =\sup _{\xi}\left\|T_{\xi} p^{\prime}\right\| \leqslant \text { const }\left\|p^{\prime}\right\|,
\end{aligned}
$$

the last inequality by 3.3 . This completes our last lemma of $\$ 4$.

We can now prove the characterization of continuously translating Lipschitz spaces that generalizes the Hardy-Littlewood characterization of $\Lambda L_{p}$. We again use the notation $\int F$ defined in the paragraph preceding 4.9.

THEOREM 4.11. For every translating space $F$, with norm $\|f\|$,

$$
\mathscr{C} \Lambda F=\int \mathscr{C} F \text {. }
$$

In more detail:

(1) $f$ is absolutely continuous with derivative $f^{\prime} \in F$, and $\left\|T_{x} f^{\prime}-f^{\prime}\right\|$ varies continuously with $x$ if and only if (2) $\left\|T_{x} f-f\right\|^{(1)}$ varies continuously with $x$.

Proof. Recall that $\left(\|f\|^{(1)}\right.$ is defined by

$$
\left.\sup _{y>0} \frac{1}{y}\left\|T_{y} f-f\right\|\right) \text {. }
$$

From 4.9 and 3.8 we know that $P \cap F$ is a dense subspace of both $\mathscr{C} \Lambda F$ and $\int \mathscr{C} F$. Furthermore, by 4.10 , these two spaces induce equivalent norms on $P \cap F$. Hence by $3.10, \mathscr{C} \Lambda F=\int \mathscr{C} F$.

\section{Characterization of continuously translating Hölder functions} $(\alpha<1)$. Given a translating space $F$, with norm $\|f\|$, and a positive number $\alpha<1$, we can define the Hölder seminorm

$$
\|f\|^{(\alpha)}=\sup _{x>0} \frac{\left\|T_{x} f-f\right\|}{x^{\alpha}}
$$

and the associated Hölder space $\Lambda^{\alpha} F$ exactly as we defined $\|f\|^{(1)}$ and $\Lambda F\left(=\Lambda^{1} F\right)$ in $\$ 2$.

Example. For $F=C$, with $\|f\|=\|f\|_{\infty}$, the space $\Lambda^{\alpha} C$ consists of the functions satisfying an ordinary uniform Hölder condition of order $\alpha$. To see that not every $f \in \Lambda^{\alpha} C$ translates continuously for the Hölder norm, take some $f \equiv x^{\alpha}$ for $0 \leqslant x \leqslant \pi$, and otherwise continuously differentiable (with mean 0 as usual). Then $f \in \Lambda^{\alpha} C$. But for all positive $x \leqslant \pi / 2$, we have

$$
\begin{aligned}
\left\|T_{x} f-f\right\|_{\infty}^{(\alpha)} & =\sup _{y} \frac{1}{y^{\alpha}}||\left(T_{x+y}-T_{x}-T_{y}+I\right) f \|_{\infty} \\
& \geqslant \sup _{y} \frac{1}{y^{\alpha}}|f(x+y)-f(x)-f(y)| \\
& \geqslant \frac{1}{x^{\alpha}}|f(2 x)-f(x)-f(x)| \\
& =\frac{1}{x^{\alpha}}\left|(2 x)^{\alpha}-x^{\alpha}-x^{\alpha}\right|=2-2^{\alpha} .
\end{aligned}
$$


By analogy with Theorem 4.11 it is natural to conjecture that $f$ translates continuously in $\Lambda^{\alpha} F$ if and only if $f$ has a continuous "fractional" derivative $f^{(\alpha)} \in \mathscr{C} F$. Yet we shall see that, in contrast with the case $\alpha=1$, this condition is sufficient but not necessary.

To describe the correct condition for continuous translation in $\Lambda^{\alpha} F$ we must look at still another associated space $\lambda^{\alpha} F$, defined by the requirement that

$$
\lim _{x=0} \frac{\left\|T_{x} f-f\right\|}{x^{\alpha}}=0 .
$$

(\| $\|$ means the original $F$ norm, as usual.) In the language of $O$ and $o, \Lambda^{\alpha} F=$ $\left\{f \in F:\left\|T_{x} f-f\right\|=O\left(x^{\alpha}\right)\right\}$ and $\lambda^{\alpha} F=\left\{f \in F:\left\|T_{x} f-f\right\|=o\left(x^{\alpha}\right)\right\}$. We shall prove in Theorem 5.8 below that for $\alpha<1$ the continuously translating subspace of $\Lambda^{\alpha} F$ is in fact $\lambda^{\alpha} F$ (while for $\alpha=1$ the space $\lambda^{1} F$ contains only the function $\equiv 0$ ).

LEMMA 5.1.

$$
\Lambda^{\alpha} F=\Lambda^{\alpha} \mathscr{C} F
$$

LEMMA 5.3.

$$
\|f\|^{(\alpha)} \geqslant\left(\frac{1}{2 \pi}\right)^{\omega}\|f\| .
$$

THEOREM 5.3. The space $\Lambda^{\alpha} F$ is complete for the norm $\|f\|^{(\alpha)}$.

The proof of the above theorem and its lemmas is exactly like the corresponding proof for $\Lambda F$ in $\S 4$.

Lemma 5.4. If $\left\|T_{x} f-f\right\|=o\left(x^{\alpha}\right)$ as $x \rightarrow 0$, then $\left\|T_{x} f-f\right\|^{(\alpha)}=o(1)$.

Proof. We can assume without loss of generality that all $T_{x}$ are isometries. By definition,

$$
\begin{aligned}
\left\|T_{x} f-f\right\|^{(\alpha)} & =\sup _{y>0}\left\|T_{y}\left(T_{x} f-f\right)-\left(T_{x} f-f\right)\right\| y^{-\alpha} \\
& =\sup _{y>0}\left\|T_{x}\left(T_{y} f-f\right)-\left(T_{y} f-f\right)\right\| y^{-\alpha} .
\end{aligned}
$$

For $o \leqslant y \leqslant x$, we have

$$
\begin{aligned}
\left\|T_{x}\left(T_{y} f-f\right)-\left(T_{y} f-f\right)\right\| y^{-\alpha} \leqslant \| T_{x}\left(T_{y} f-f\right) & \| y^{-\alpha} \\
& +\left\|T_{y} f-f\right\| y^{-\alpha}=2\left\|T_{y} f-f\right\| y^{-\alpha} .
\end{aligned}
$$

And for $y \geqslant x \geqslant 0$ we have $\left\|T_{y}\left(T_{x} f-f\right)-\left(T_{x} f-f\right)\right\| y^{-\alpha} \leqslant\left\|T_{y}\left(T_{x} f-f\right)\right\|$ $x^{-\alpha}+\left\|T_{x} f-f\right\| x^{-\alpha}=2\left\|T_{x} f-f\right\| x^{-\alpha}$. Hence

$$
\left\|T_{x} f-f\right\|^{(\alpha)} \leqslant 2 \sup _{0 \leqslant y \leqslant x}\left\|T_{y} f-f\right\| y^{-\alpha},
$$

from which inequality the lemma follows immediately. 
Lemma 5.5. $\quad \lambda^{\alpha} F$ is a closed subspace of $\Lambda^{\alpha} F$.

Proof. Let $f_{n} \rightarrow f$ in $\Lambda^{\alpha} F$, with $f_{n} \in \lambda^{\alpha} F$. Choose $f_{n}$ such that $\left\|f-f_{n}\right\|^{(\alpha)}<\epsilon$. Writing

$$
A_{n}=\frac{T_{x}-I}{x^{\alpha}}
$$

we have $\left\|A_{x}\left(f-f_{n}\right)\right\|<\epsilon$ for all $x$. We want $\left\|A_{x} f\right\| \rightarrow 0$. And we have $\left\|A_{x} f\right\| \leqslant\left\|A_{x}\left(f-f_{n}\right)\right\|+\left\|A_{x} f_{n}\right\|$. Hence

$$
\lim _{x \rightarrow 0}\left\|A_{x} f\right\| \leqslant \epsilon+\lim _{x \rightarrow 0}\left\|A_{x} f_{n}\right\|=\epsilon .
$$

Since $\epsilon$ was arbitrary, then

$$
\lim _{n \rightarrow 0}\left\|A_{x} f\right\|=0
$$

and $f \in \lambda^{\alpha} F$, proving the lemma.

Lemma 5.6. $\left\|T_{x} p-p\right\|=o\left(x^{\alpha}\right)$ for every trigonometric polynomial $p \in F$.

Proof. By 4.7,

$$
\frac{\left\|T_{x} p-p\right\|}{x}
$$

converges to $\left\|p^{\prime}\right\|$. Hence

$$
\frac{\left\|T_{x} p-p\right\|}{x^{\alpha}}=\frac{\left\|T_{x} p-p\right\|}{x} x^{1-\alpha} \rightarrow o \text { as } x \rightarrow o .
$$

Lemma 5.7. If $\left\|T_{x} f-f\right\|^{\alpha}=o(1)$ as $x \rightarrow 0$, then $\left\|T_{x} f-f\right\|=o\left(x^{\alpha}\right)$.

Proof. The lemma asserts that $\mathscr{C} \Lambda^{\alpha} F \subseteq \lambda^{\alpha} F$. If $f \in \mathscr{C} \Lambda^{\alpha} F$, from 3.8 it follows that $f$ is the limit in $\Lambda^{\alpha} F$ of trigonometric polynomials $p_{n}$. By 5.6 each $p_{n} \in \lambda^{\alpha} F$, and by $5.5 \lim p_{n}=f \in \lambda^{\alpha} F$.

The conjunction of Lemmas 5.4 and 5.7 is

TheOrem 5.8. For every translating space $F, \mathscr{C} \wedge^{\alpha} F=\lambda^{\alpha} F$.

In other words, the following functions must both be continuous or both be discontinuous for $x \geqslant 0$ :

$$
\begin{aligned}
& x \rightarrow\left\|T_{x} f-f\right\|^{(\alpha)}, \\
& x \rightarrow\left\|T_{x} f-f\right\| x^{-\alpha},
\end{aligned}
$$

the norm $\|f\|^{(\alpha)}$ being defined as

$$
\sup _{y>0}\left\|T_{y} f-f\right\| y^{-\alpha} \text {. }
$$

Finally, to justify the remark about fractional derivatives in the third paragraph of this section, it will be convenient to use Zygmund's smooth functions (6), which by definition (are continuous and) satisfy

$$
\left\|T_{x} f+T_{-x} f-2 f\right\|_{\infty}=o(x) .
$$


As usual, $\|f\|_{\infty}=\sup _{y}|f(y)|$. Let us write $\lambda^{*} C$ for the space of smooth functions. (One can of course define $\lambda^{*} F$ for any translating space $F$, and also $\Lambda^{*} F$ with $O$ replacing $o$.) Let us write $\int{ }^{(\alpha)} f$ for the Weyl fractional indefinite integral of $f$, defined to have Fourier series $\sum(\text { in })^{-\alpha} c_{n} e^{i n x}$ when $f$ has Fourier series $\sum c_{n} e^{i n x}$. Zygmund (6 Theorems 11 and 12, p. 53) proves that $\int(\alpha) f \in \lambda^{\alpha} C$ if and only if $\int f \in \lambda^{*} C$. It follows that if $f$ is continuous then $\int(\alpha) f \in \lambda^{\alpha} C$, since every continuously differentiable function belongs to $\lambda^{*} C$. On the other hand, to demonstrate the existence of discontinuous $f$ with $\int{ }^{(\alpha)} f \in \lambda^{\alpha} C$, it is enough to exhibit an $f \in \lambda^{*} C$ that is not continuously differentiable. But in fact Zygmund shows there are smooth $f$ that fail to have a derivative at almost all points.

\section{REFERENCES}

1. S. Bochner, Additive set functions on groups, Ann. Math., 40 (1939), 769-799.

2. K. de Leeuw, Linear spaces with a compact group of operators, Ill. J. Math., 2 (1958), 367-377.

3. G. H. Hardy and J. E. Littlewood, Some properties of fractional integrals I, Math. Zeit., 27 (1927-1928), 565-606.

4. A. Plessner, Eine Kennzeichnung der totalstetig Funktionen, J. reine angew. Math., 160 (1929), 26-32.

5. G. E. Silov, Homogeneous rings of functions, Uspehi Matem. Nauk N.S., 6 (1951), A.M.S. Translation 92 .

6. A. Zygmund, Smooth Functions, Duke Math. J., 12 (1945), 47-76.

Dartmouth College 